\title{
Crystal structures of amino acids: from bond lengths in glycine to metal complexes and high pressure polymorphs
}

Carl Henrik Görbitz

Department of Chemistry, University of Oslo, N-0315 Oslo, Norway

$\underline{\text { Schematic illustrations of amino acids in the Cambridge Structural Database identified by their refcodes. }}$

For organic structures the following applies to the listed refcodes:

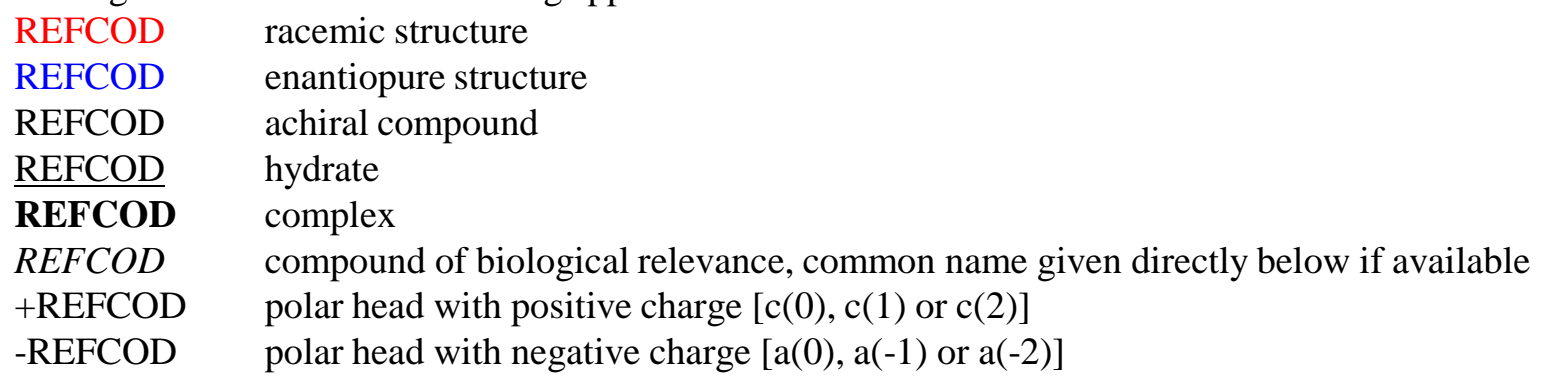

If a substance has been crystallized in different states, the illustration will appear under $\mathrm{z}(0)$ (if applicable). For metalloorganic structures other font codes apply, see page 18.

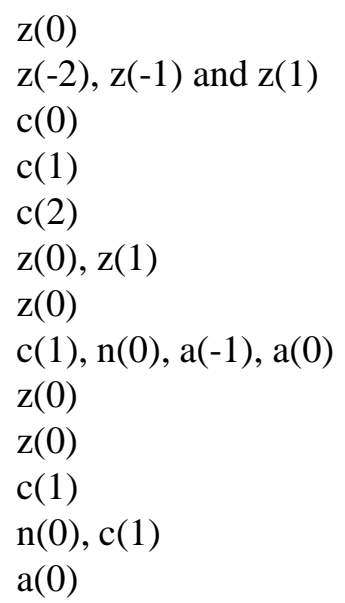

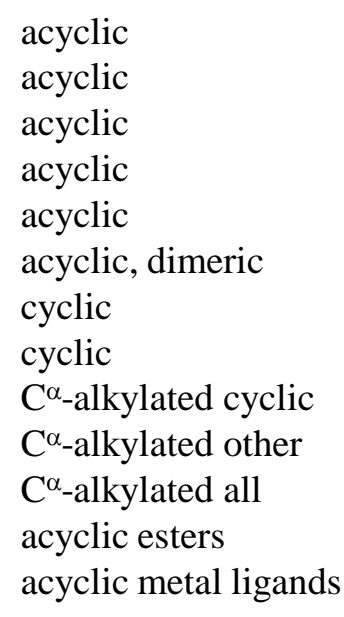

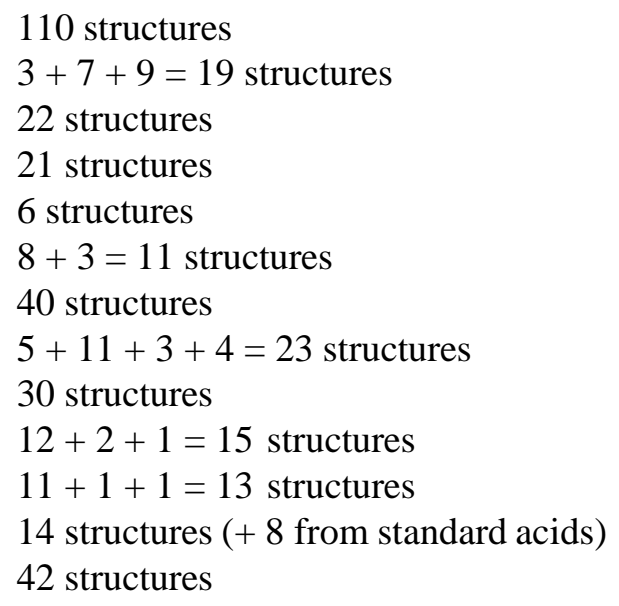


$z(0)$

GEFHEF

GEJCAA

GEJLEN

HUWSOI

HUWSOI01

+ZAPCOK

\section{DLABUT02}

DLABUT03

DLABUT05

DLABUT12<smiles>O=c1[nH]c(=O)n(CO)cc1Br</smiles>

BADNAV

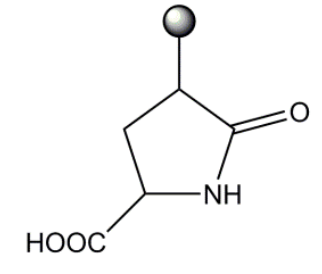

ACXMPR
$\mathrm{COOH}_{\mathrm{CO}}$

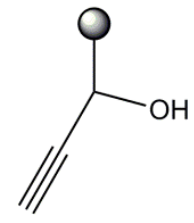

AMMALA<smiles>O=C(O)C(O)F</smiles><smiles>CCC1CS(=O)(CCO)=NC1=O</smiles><smiles>NC(N)=NOCCO</smiles>

BEYFAM canavanine $+\underline{\text { HIVSUA }}$
○

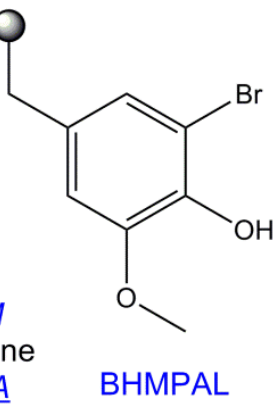

ATIVOO

ATIWAB

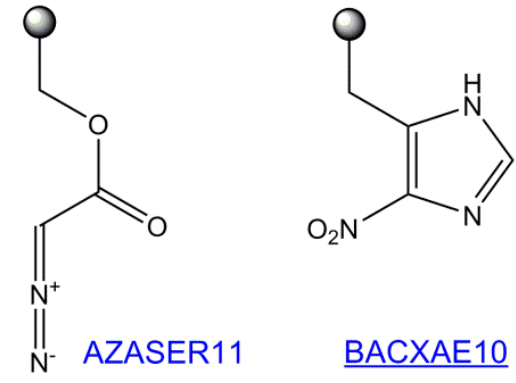

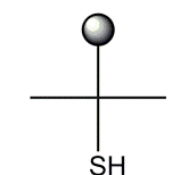

CEDFAS penicillamine +PENILA10

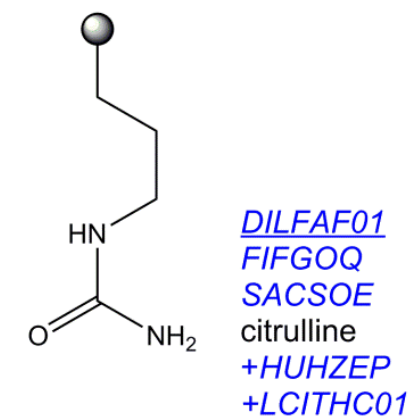

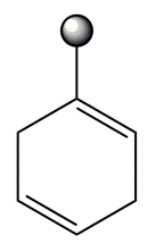

CERGEL DEYFOD +MAJTAT

CHEDGL10

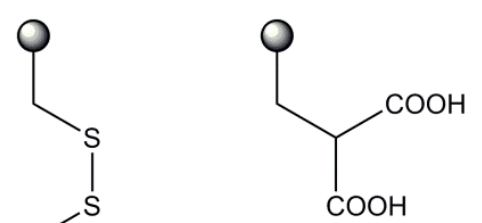

CUVFOO

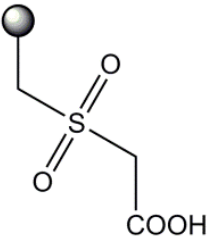

CXMCYS

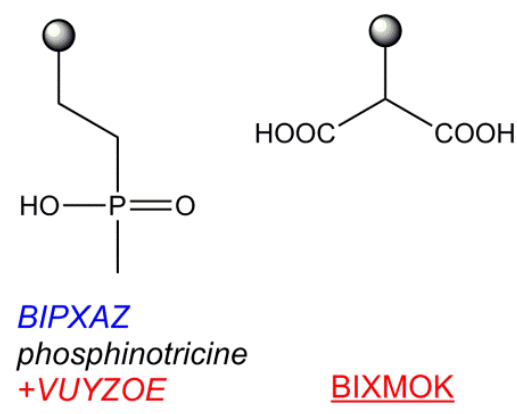

BUHGOA01 homoserine

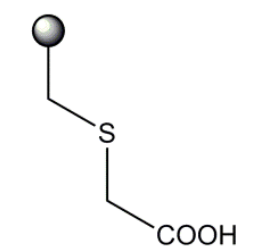<smiles>O=c1[nH]cc(SCO)c(=O)[nH]1</smiles>

CXMCYT CYRSUC10<smiles>NC(=O)CCSCO</smiles><smiles>CCCCCO</smiles><smiles>O=Cc1ccc(B(O)O)cc1</smiles><smiles>O=CN(NO)[N+](=O)O</smiles><smiles>OCc1ccccc1O</smiles>

LNLEUC10

DNLTYR10

DOXXAP alanosine

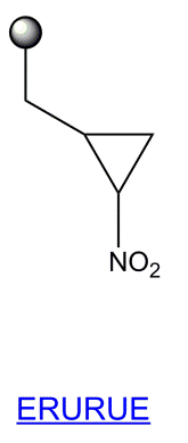

DTYROS +OTROSC 
${ }^{+} \mathrm{H}_{3} \mathrm{~N}$<smiles>OCc1ccc(F)cc1CO</smiles><smiles>OCc1cc(F)c(F)c(F)c1</smiles>

EXAXAC EXAXEG<smiles>CS(=N)(=O)CCO</smiles>

FADVUB METTSO10

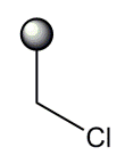

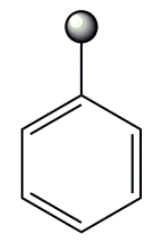

EXAXOQ
FIVGEW

+ CABVUX

+ FAPMIT

+ FAPMOZ

+ +GACVIP
+ HAZGIZ

+ IROVEQ

+ NILXUB

+ RENKUQ

+TEHYUA

+ WIHJII

+XALCOC01

+ XALCUI

+ XALDAP
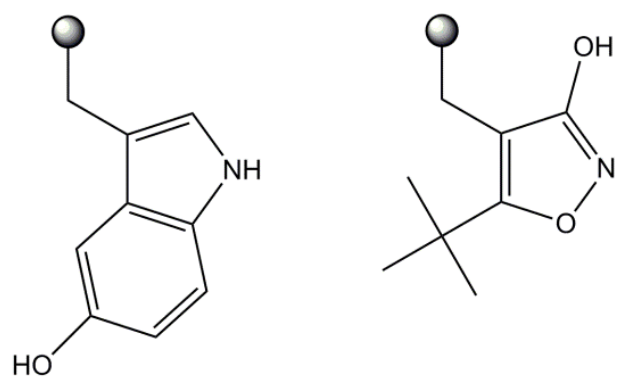

\section{HTRYPT10 \\ MUYTEG \\ MUYTOQ \\ MUYVAE}

HUKFUO
HMEBUT

HMFGLA
HMGLUT

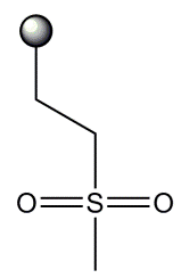

IMIWUW<smiles>CN(C)C1SC(C(O)O)C(O)C1O</smiles><smiles>OCSCC(F)(F)F</smiles>

JORDID JOXBED

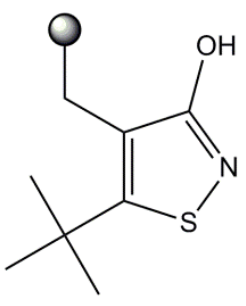

HUKGAV*

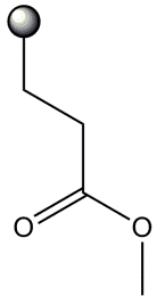

GAVRAX<smiles>CC1(C)OCC(C(O)O)O1</smiles>

ICAMOO 


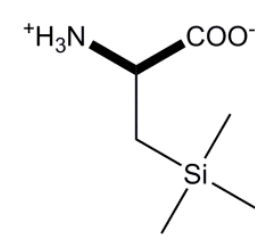

(C)

KUKMAF

KURCOQ<smiles>OCc1cc(O)c(O)cc1F</smiles>

LOZPAR<smiles>O=Cc1cccc(O)c1</smiles>

MTYROS01

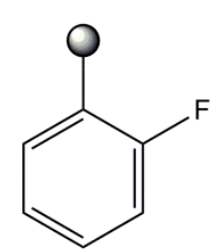

LALXAX<smiles>CC(C)C(O)CO</smiles>

MATVIM<smiles>Oc1cc(O)on1</smiles>

OBEKUB

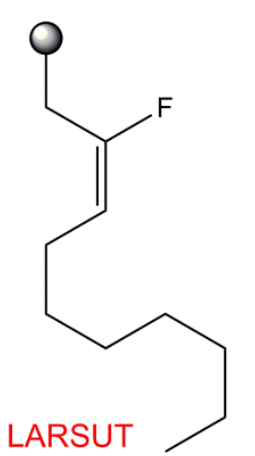<smiles>O=Cc1ccc(O)c(O)c1</smiles><smiles>OCc1cc(O)c(O)cc1F</smiles>

LEQTEG
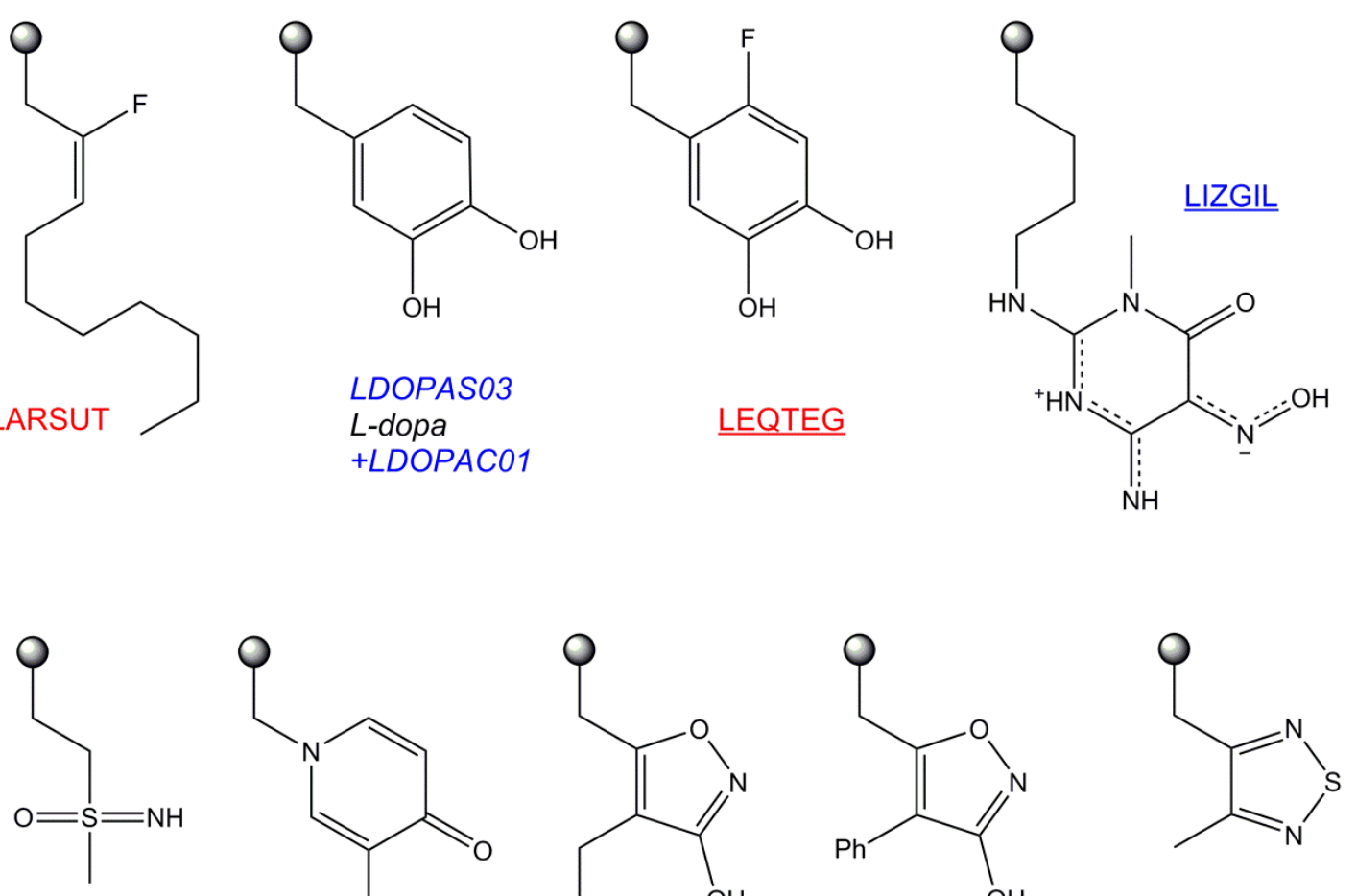<smiles>O=c1ccn(CO)cc1O</smiles><smiles>CCCc1c(O)noc1CO</smiles><smiles>OCc1onc(O)c1-c1ccccc1</smiles>

METTSO10

MIMOSN mimosine + LMIMOS<smiles>OCSCc1ccncc1</smiles>

OCAZOI

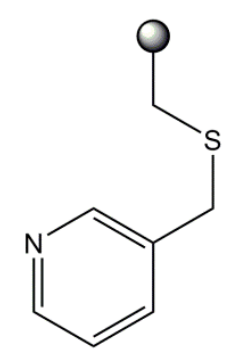

OCAZUO

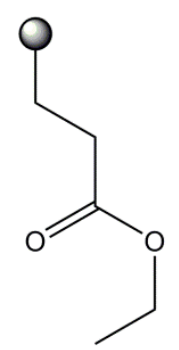

PAZHEE

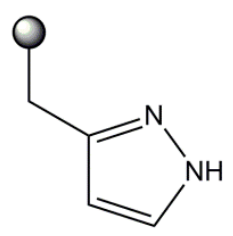

PYRZAL10

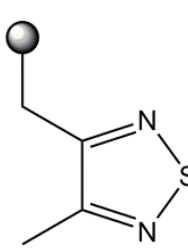

MOFGOD

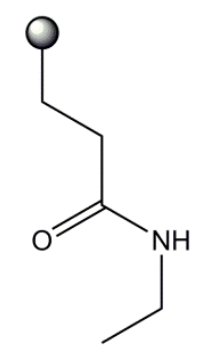

QAJXOQ 
<smiles>N[C@@H](Cn1oc(=O)[nH]c1=O)C(=O)O</smiles>

QUIZAL quisqualic acid

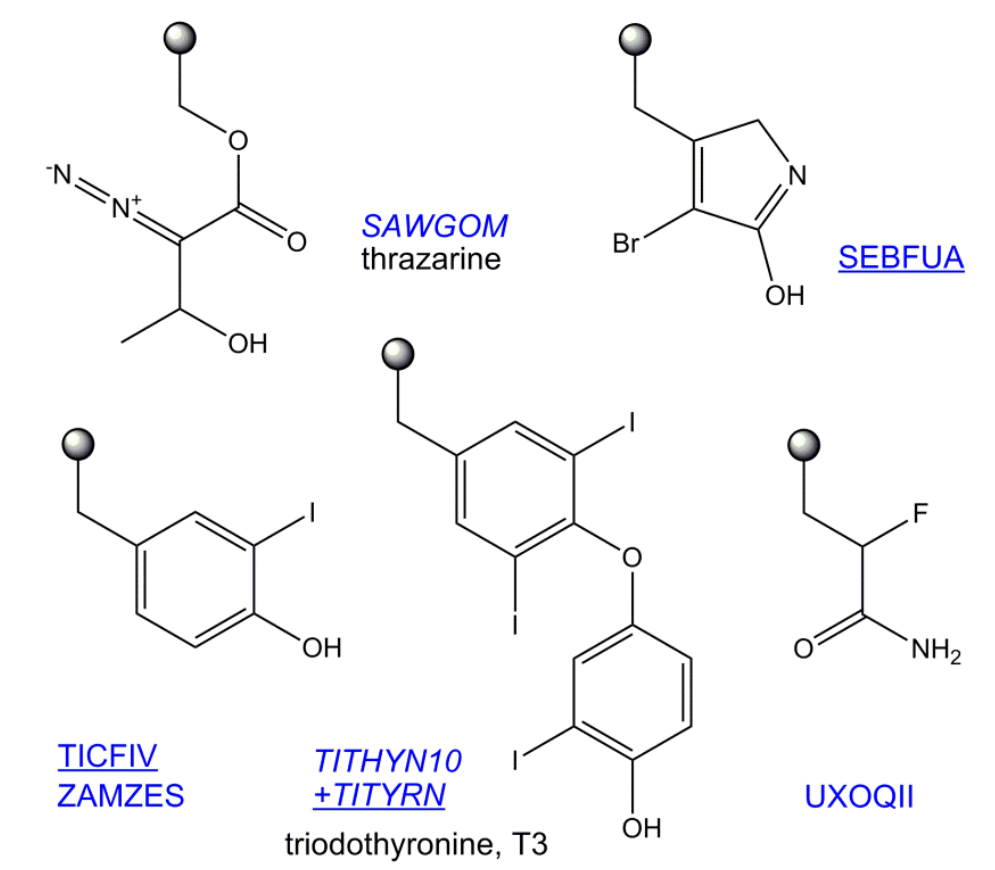

${ }_{\mathrm{OH}}^{\mathrm{O}}$

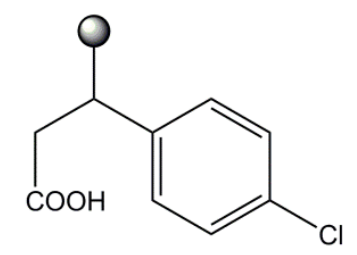

RAYWIX

RAYWOD<smiles>Oc1ccccc1P(c1ccccc1)c1ccccc1</smiles>

RIJFOF<smiles>OCC(F)CO</smiles><smiles>CC(C)(C)CO</smiles><smiles>O=[N+]([O-])c1ccc(CO)cc1</smiles><smiles>Cc1onc(O)c1CO</smiles><smiles>OCC/N=C/c1ccccc1O</smiles>

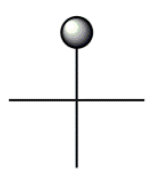

SIMPOT<smiles>OC(C(F)(F)F)C(F)(F)F</smiles>

SIMRAH<smiles>CS(=O)CO</smiles>

SMECYO

SAQQIK

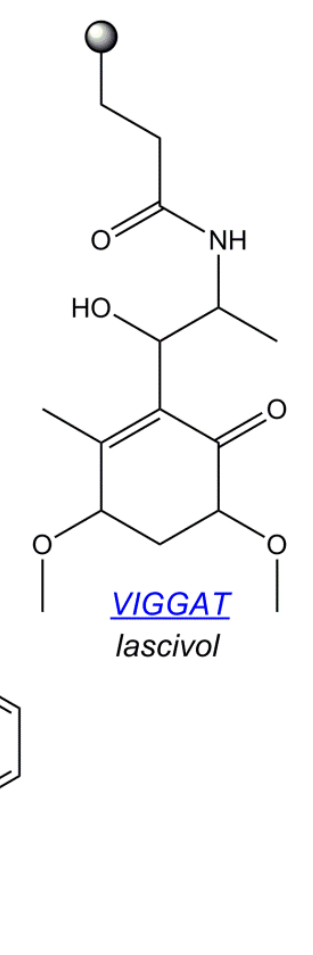<smiles>O=C(O)c1cccs1</smiles>

THDSER

SAQQIK
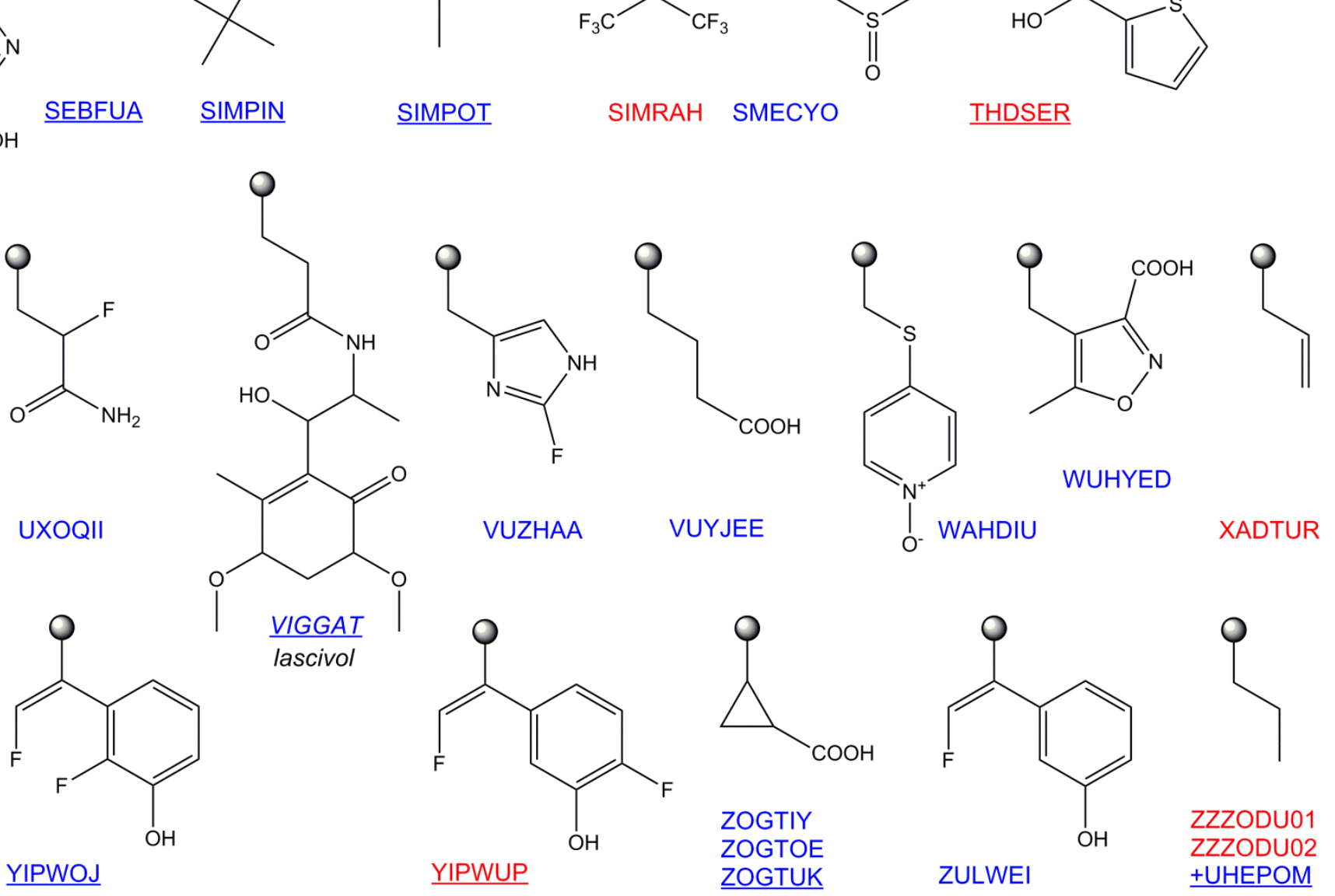
$z(-2)$
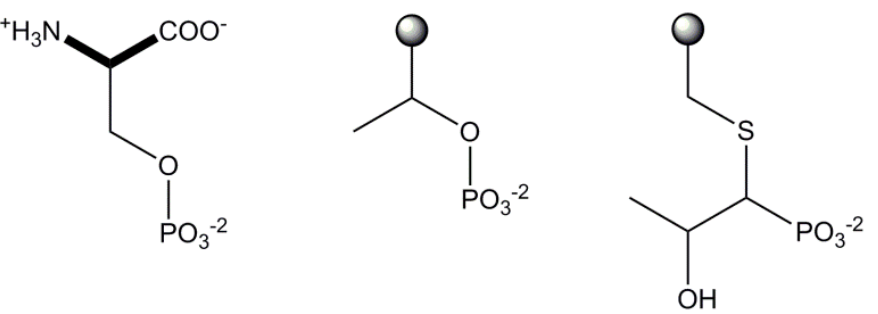

ATEBAC

ATEBIK

ATEBIK01

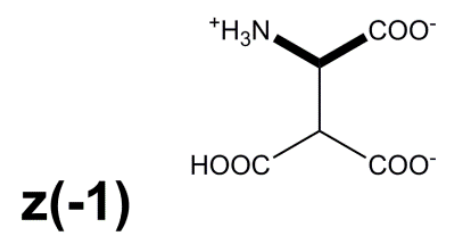

$z(1)$

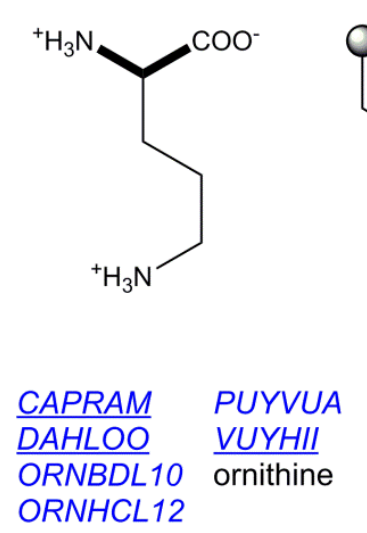

GEJKUC

IJICIN $z(-1)$

ACXGLU

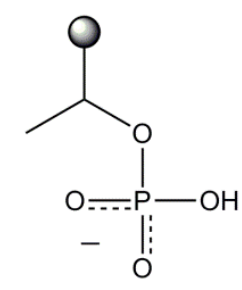

\section{GEDPIP}

IJICAF

IJICEJ

+POTHRE01

+THREPO0 1

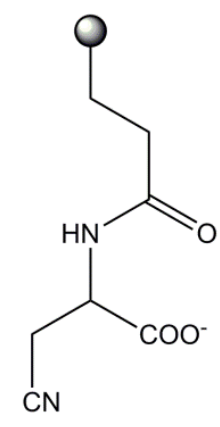

HIBZUN

CXMETN10
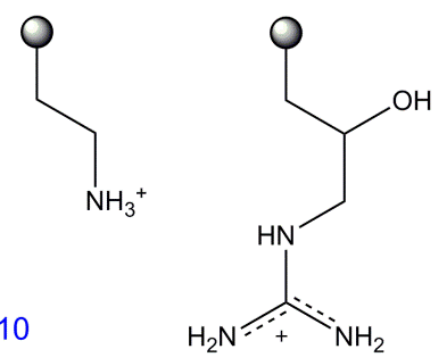

DABUTA01

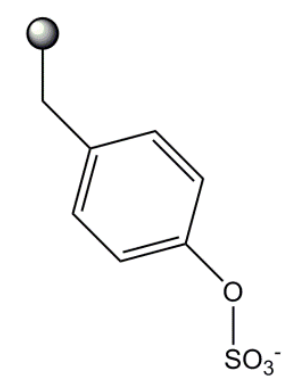<smiles>O=S(=O)(O)CO</smiles>

KTYSUH10

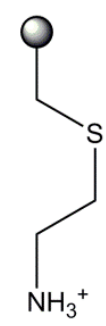

JINGOC

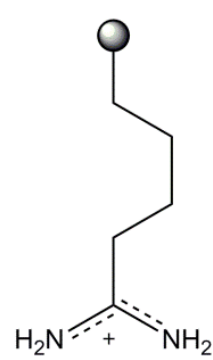

KUFCIX indospicine

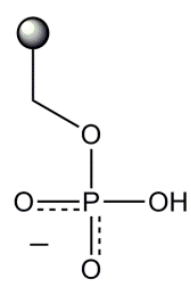

LEPNAV* + SERIPH 10 +SERPOP01

LAKWEZ<smiles>[NH3+]CC(O)CCO</smiles>

ROLCOL

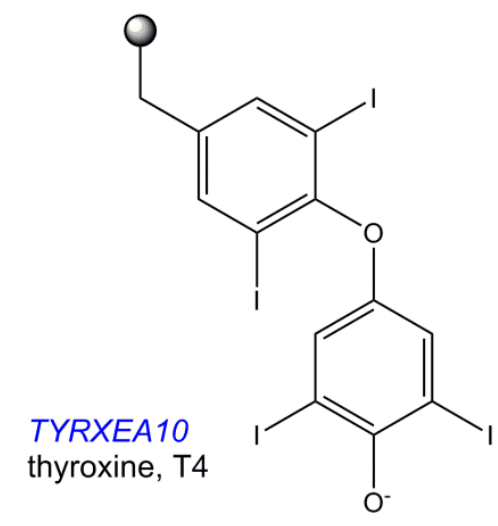<smiles></smiles><smiles>C[NH2+]CO</smiles>

SONSUK SONSUK01
YAKVOV 


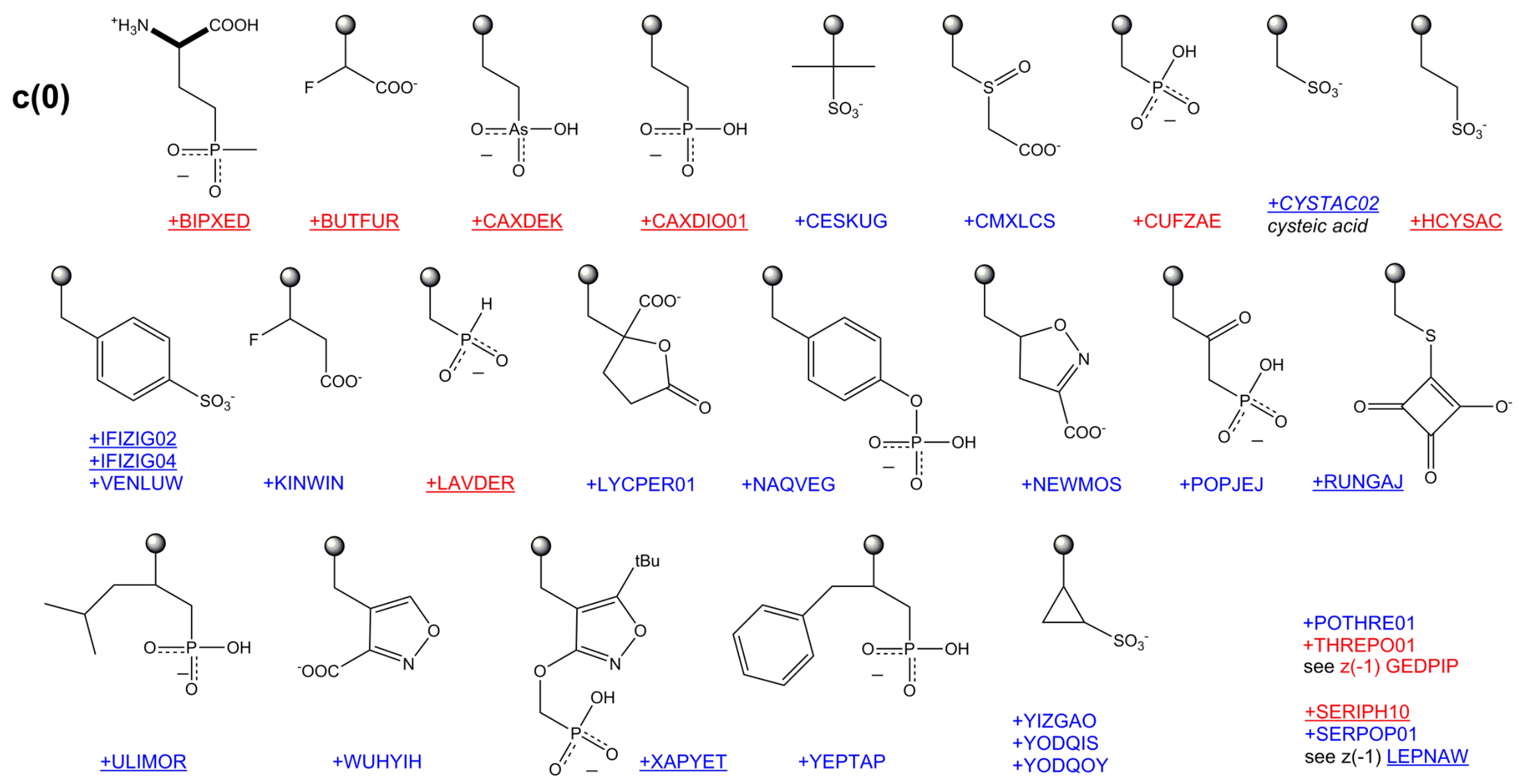




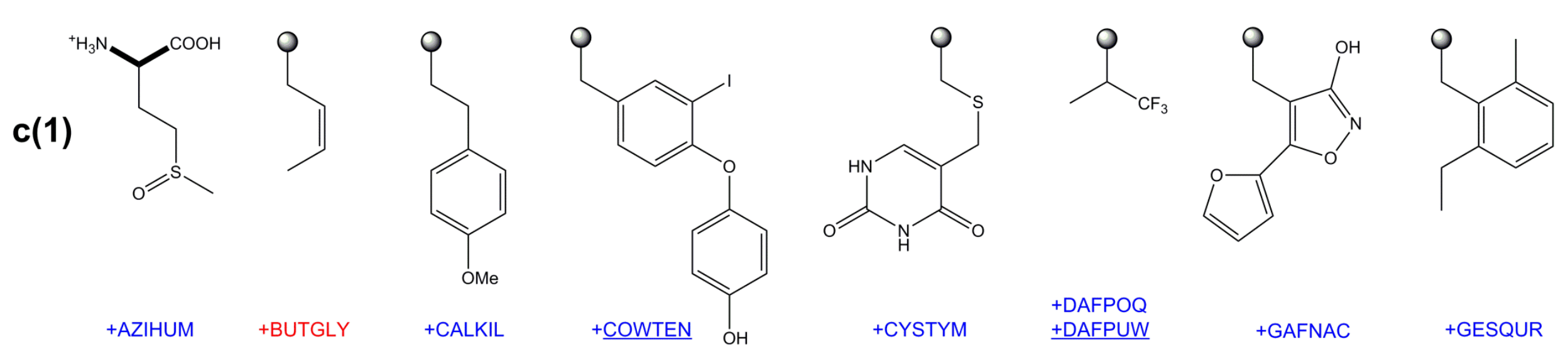

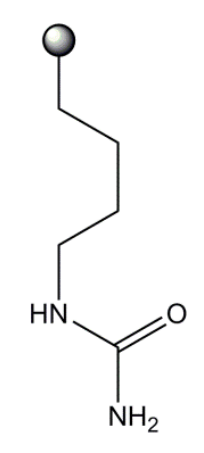

+ HCITUL<smiles>COc1c(Br)cc(CO)cc1Br</smiles>

+MAFYOI
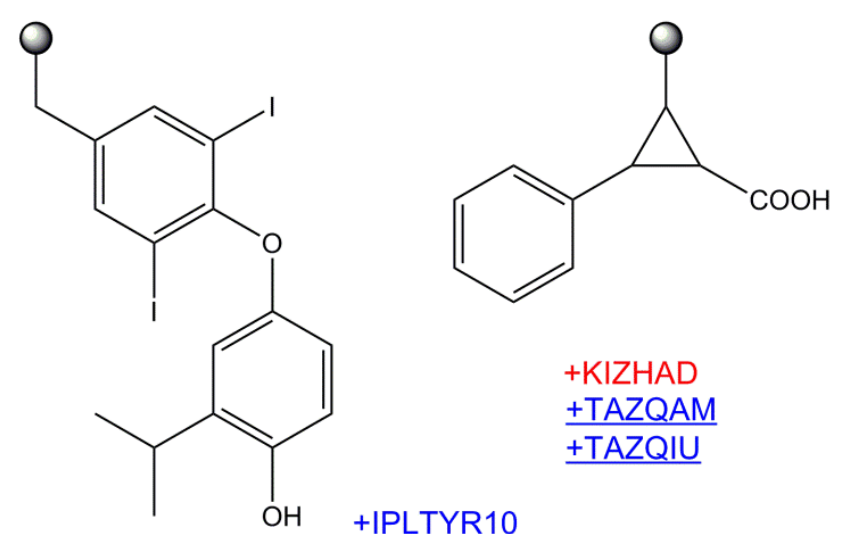

$$
\begin{aligned}
& \text { +KIZHAD } \\
& + \text { +TAZQAM } \\
& \hline \text { +TAZQIU }
\end{aligned}
$$

+IPLTYR10<smiles>O=CCCCB(O)O</smiles>

+NITFOL

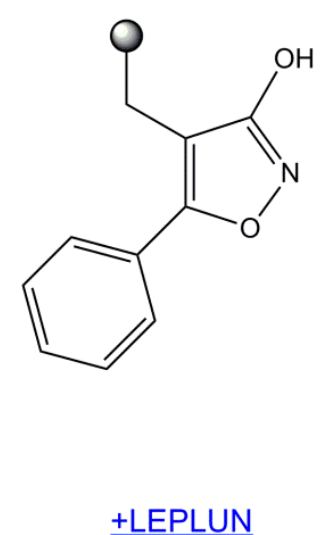<smiles>O=[N+]([O-])c1ccc(C(O)O)cc1</smiles>
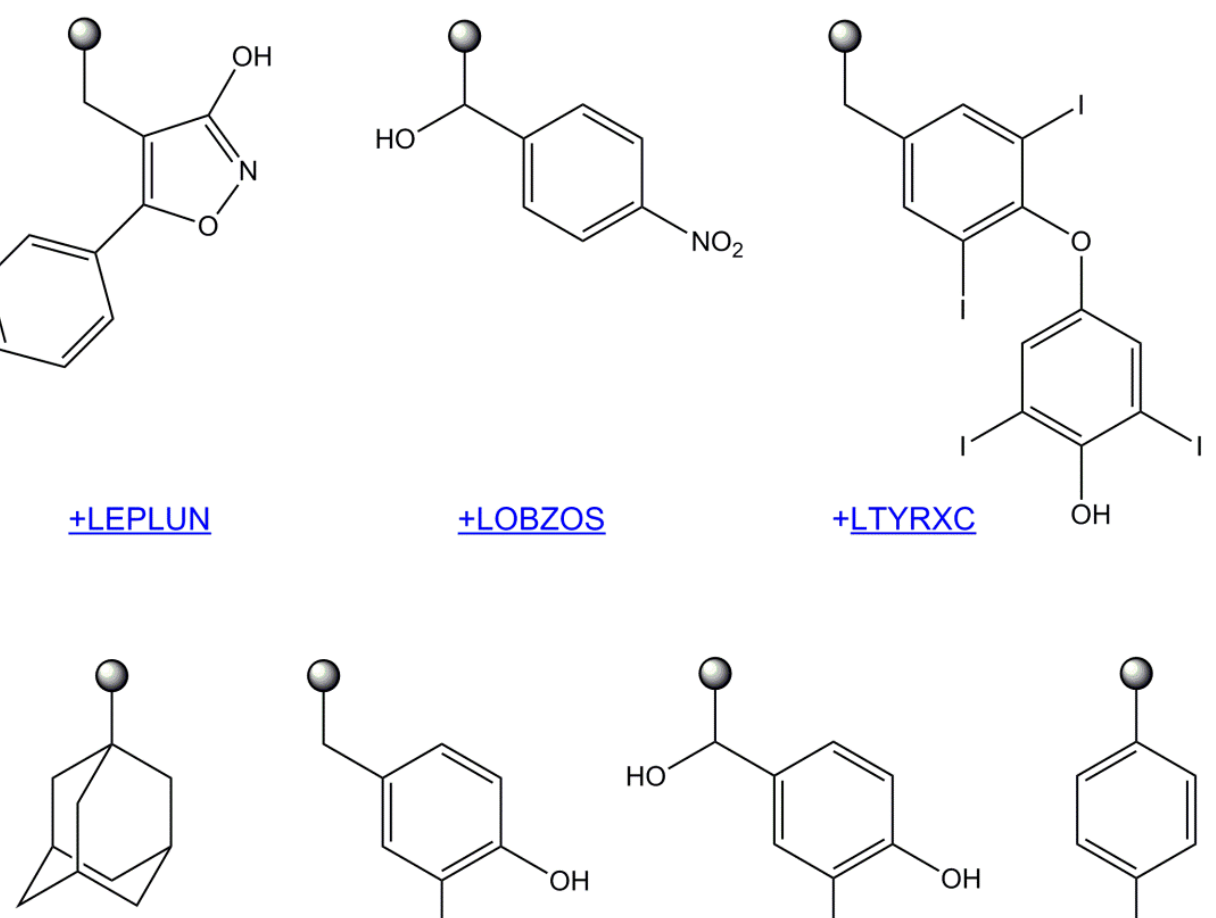<smiles>O=Cc1ccc(O)c([N+](=O)[O-])c1</smiles><smiles>O=C(O)c1ccc(O)c(O)c1</smiles>

+XPSERC<smiles>Oc1ccc(O)cc1</smiles>

+ZIGJII +ZIGJOO 


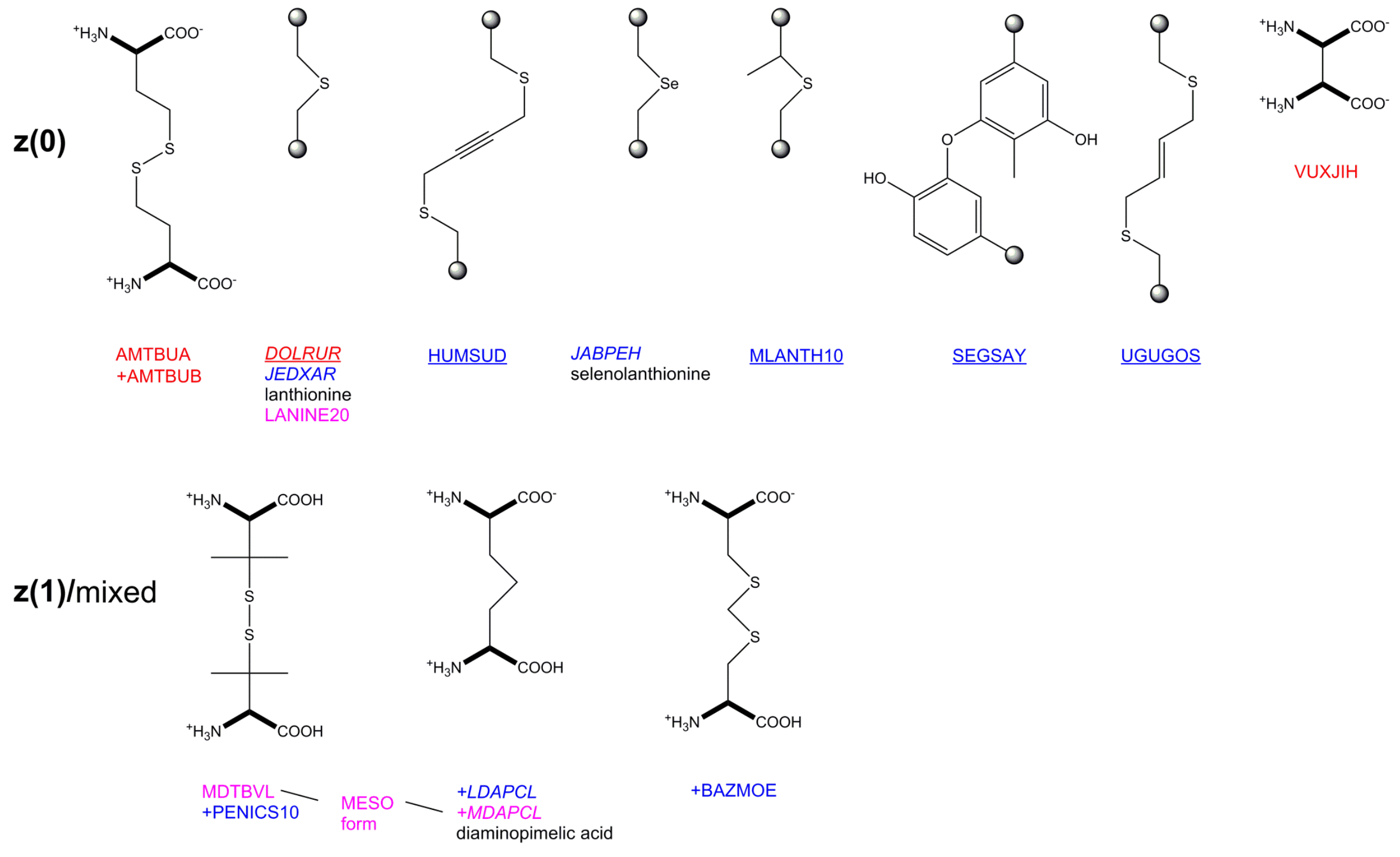


$z(0)$
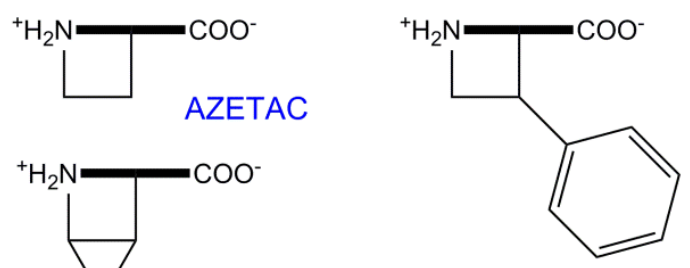

AZPCOH MATVAE

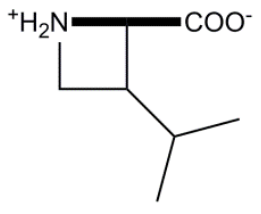

MATVEI

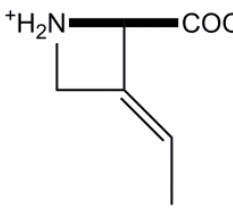

WAVKIN

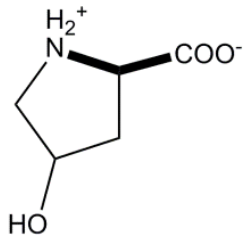

AHLPRO

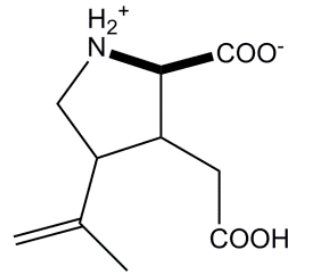

ALKINA01

allo-kainic acid

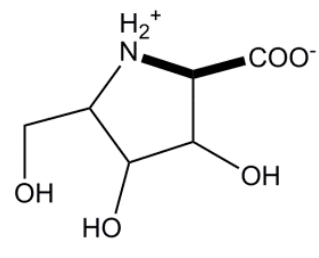

CUDCAG

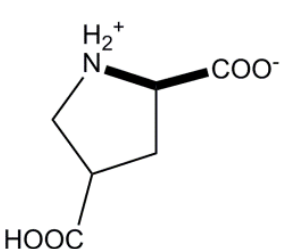

CXPROL

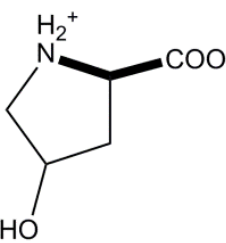

HOPROL12 -CAPROL10

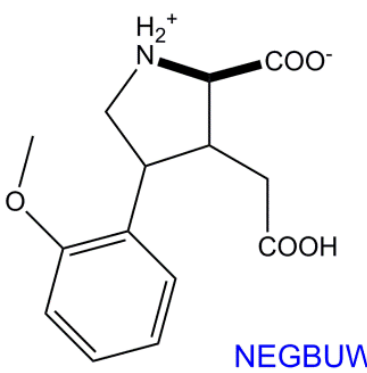

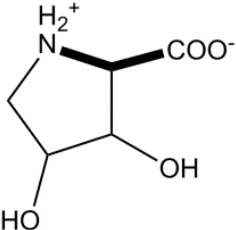

DHPROL10 FIZDOH

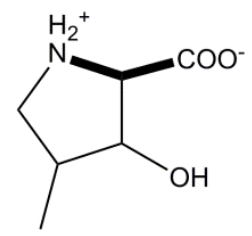

HXMPRO

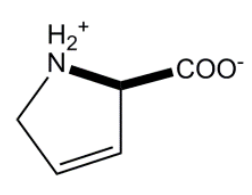

DHPROX

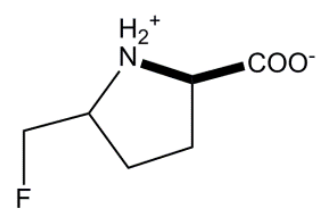

EQIHUI

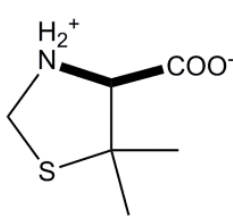

FOWLAE*

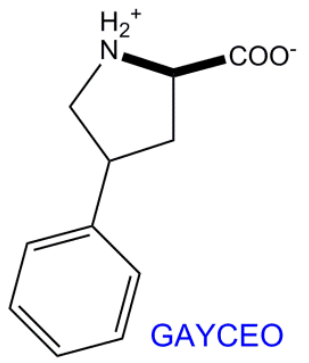

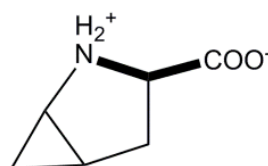

GEVMOK

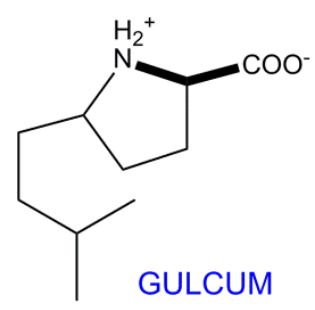

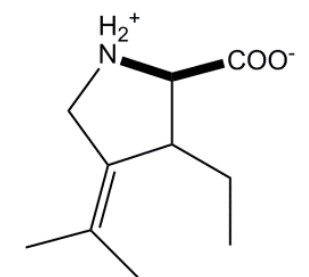

KAINAH

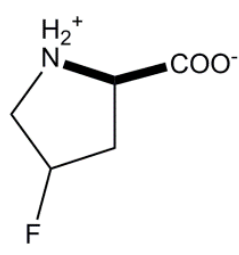

LEGBEG

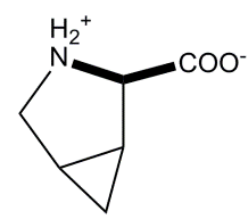

\section{MPROLT}

+MPROLC

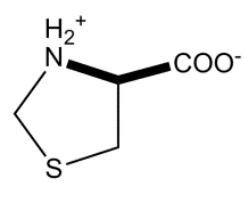

NELSEC

TASOLC02

+TPROCL
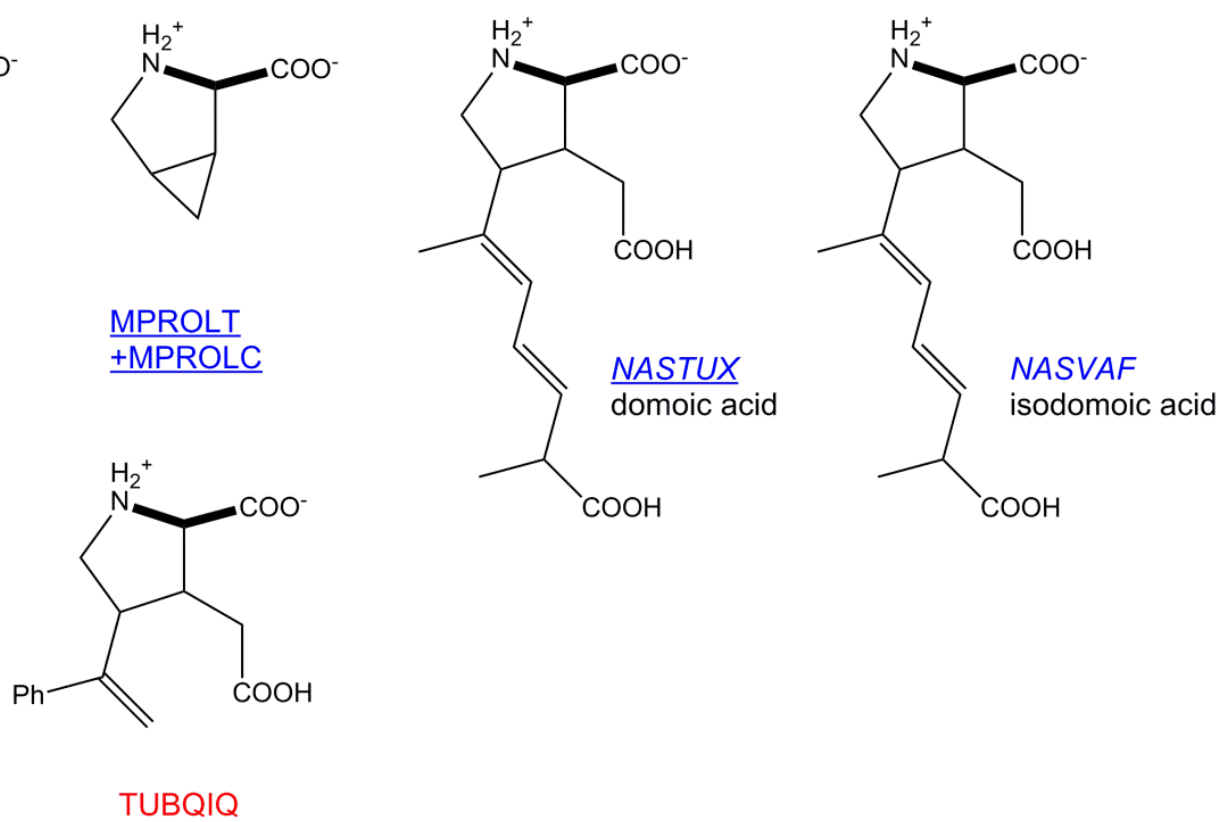

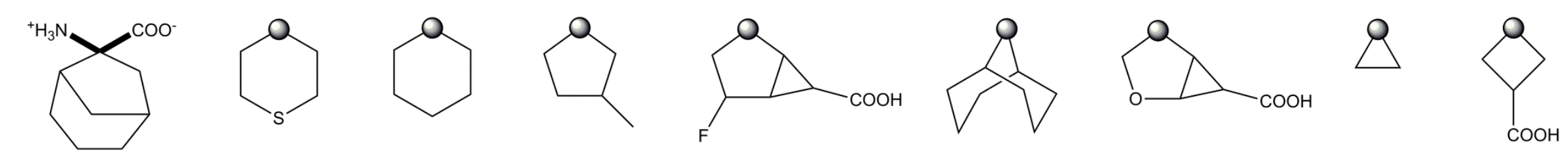

\section{$\underline{\mathrm{ABCOCK}}$}

ACXTPY

ACYHXA01 AMCPCA10

BETCUS

CIHMUB

COKJER

FOBJUB

GEHBIE01 MEGLAC
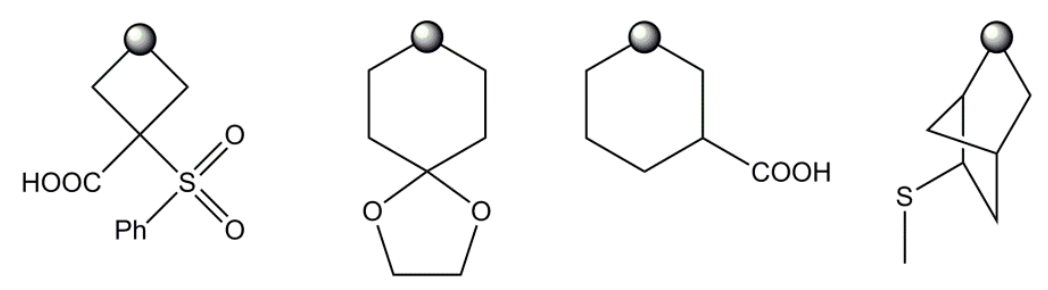

GEHBOK

JAPJUF

JUXKES
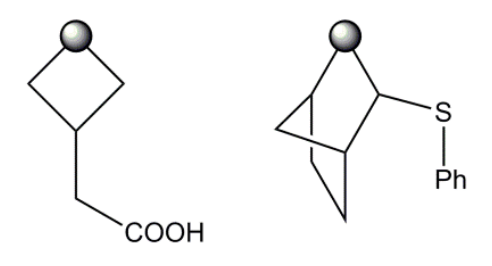

$\underline{\mathrm{KIDHOU}}$

$$
\begin{aligned}
& \text { KEPJOE } \\
& \text { PACWOF }
\end{aligned}
$$
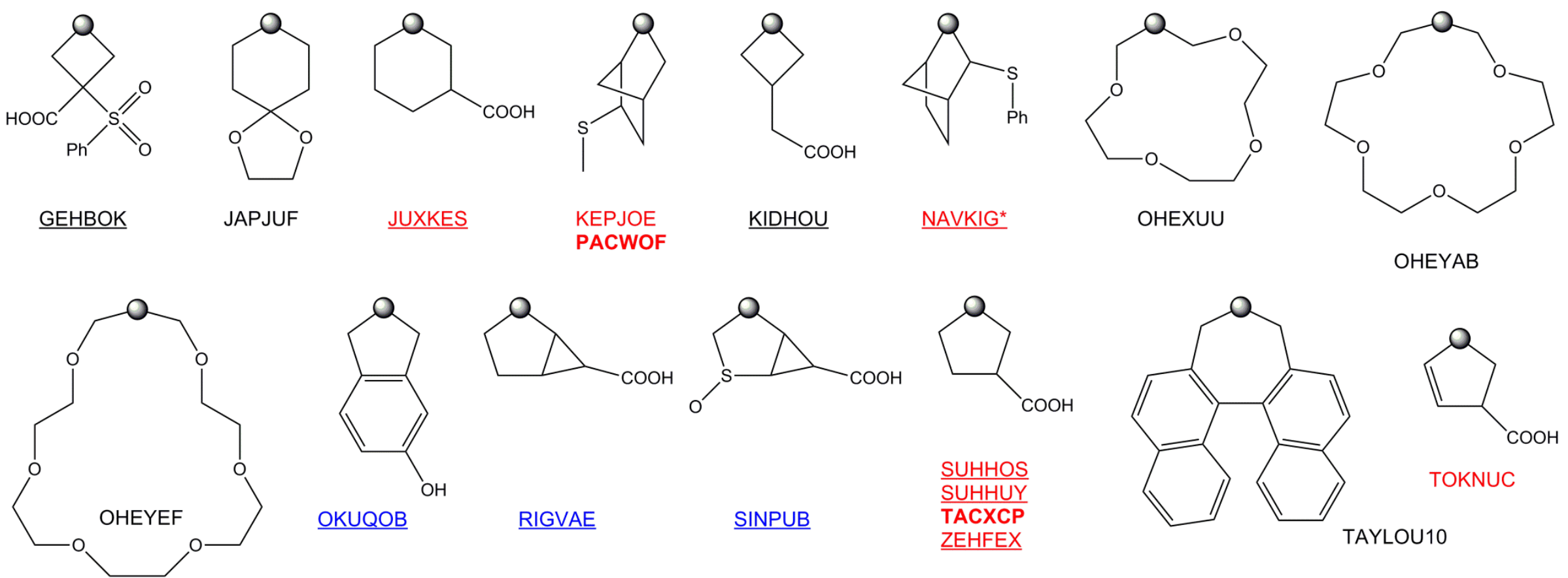

TACXCP ZEHFEX

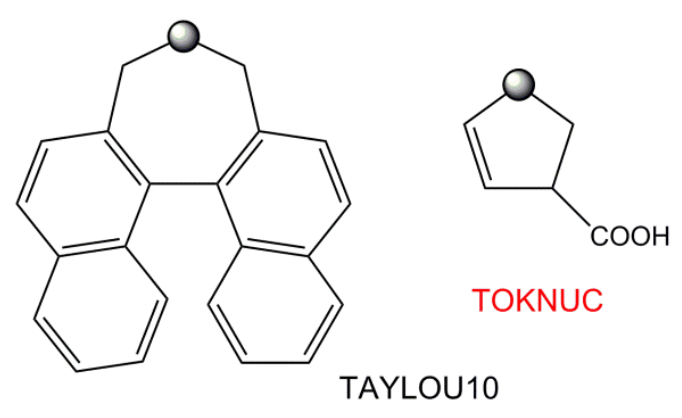

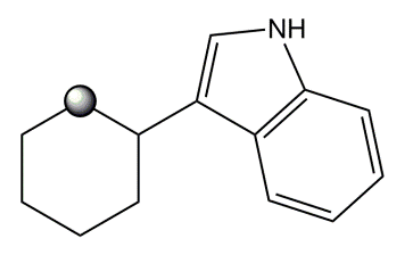

UHAWUV
YOFHEG

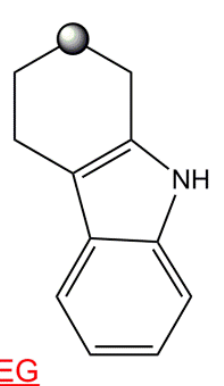

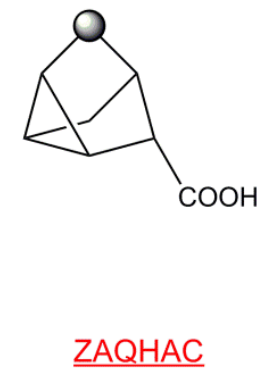

YUMHUJ

ZAQHAC
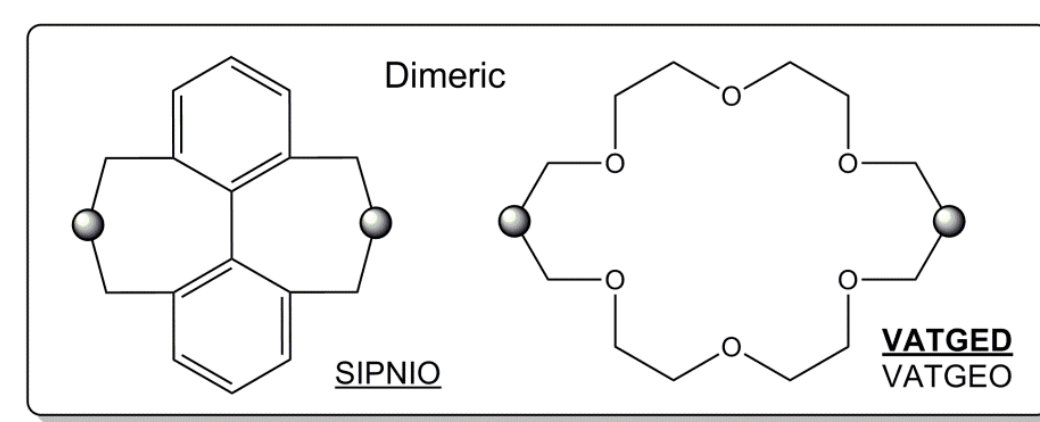


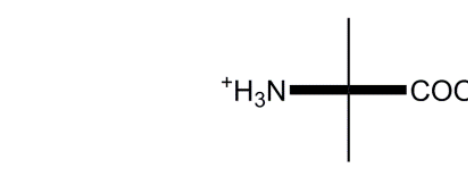

$z(0)$

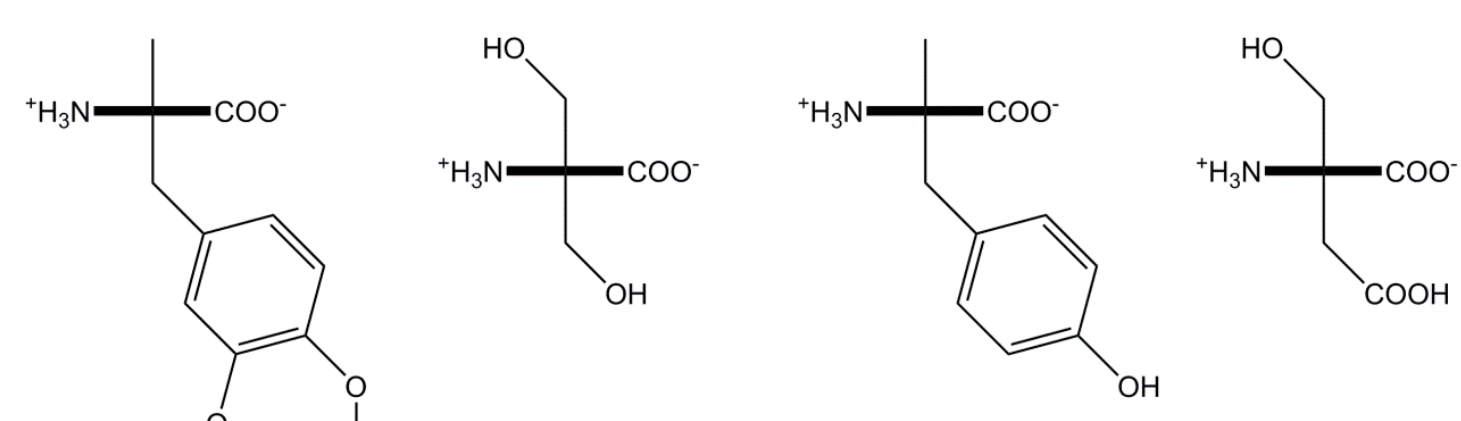

AMMPRA01

+HOTHII

+KENDOW
CITZIO10

COSGUM

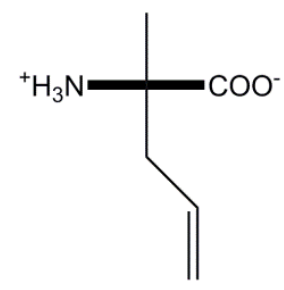

OBAYOF

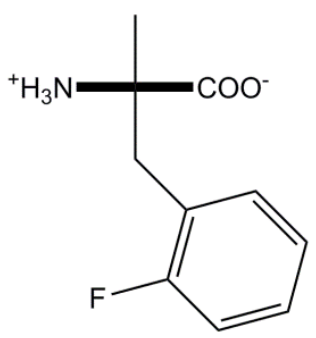

QORRIY

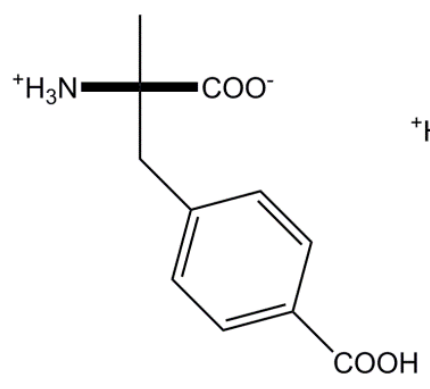

REMVEK

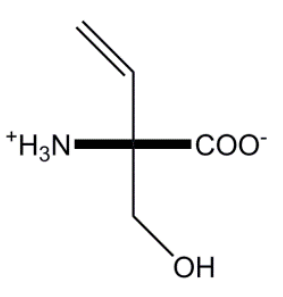

SARBUI

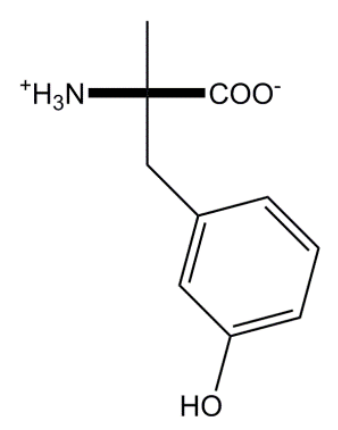

MEMTYR10

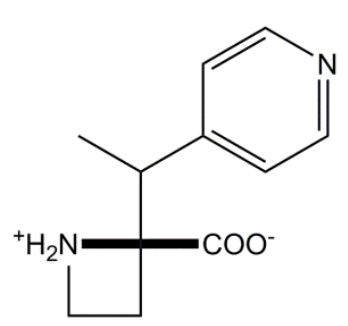

TACLEO

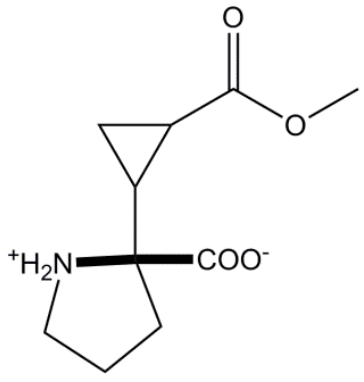

ZOCJOQ

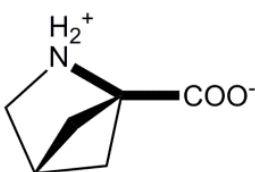

MEPROL

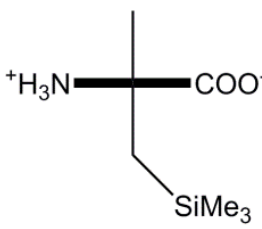

XUPGIZ 


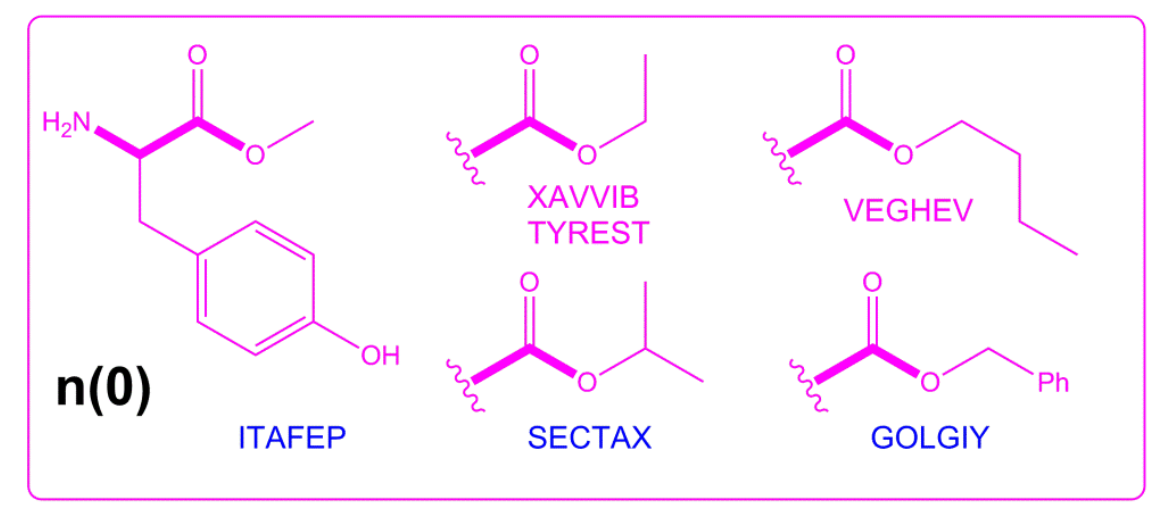

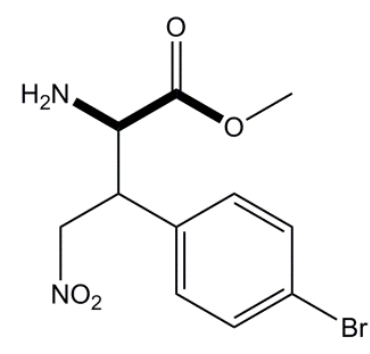

FACYEP
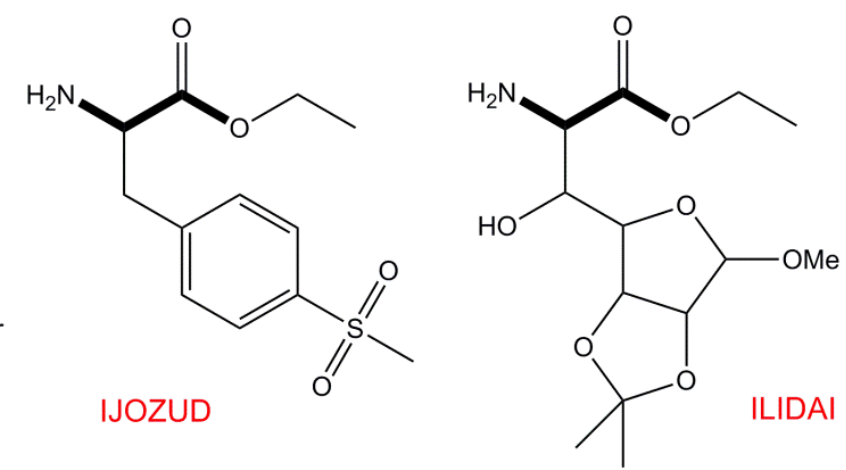<smiles>COC(=O)[C@H](N)Cc1cc(C)c(C)c(I)c1</smiles>

ITHYRM10
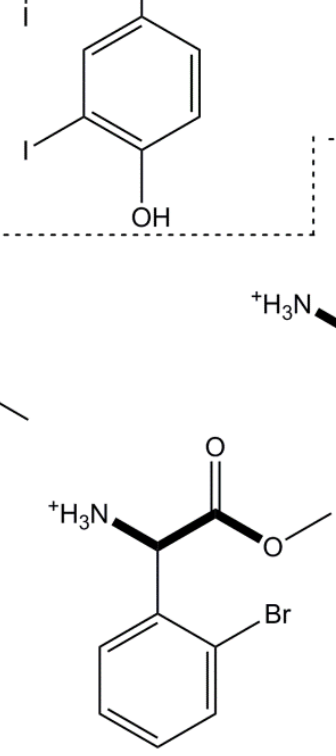

CUQVEQ<smiles>CCOC(=O)P(=O)(OCC)C(C(=O)OC)C(c1ccccc1)C([C@@H](N)C(=O)OC)P(=O)(OC)OCC</smiles><smiles>CC1(OC(=O)[C@H](N)Cc2ccccc2)Cc2ccsc2C1=O</smiles>

POQDUV

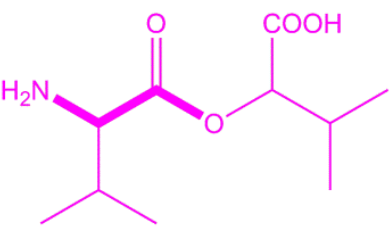

VAHIVO<smiles>CC(C)C1CCC2(C)CC(OC(=O)[C@H](C)N)C3CCC12O3</smiles>

DAUCYL10<smiles>CC(C)OC(=O)[C@@H](N)C1CCCC(O)C1</smiles><smiles>CCOC(=O)C(N)C(=O)OCC</smiles>

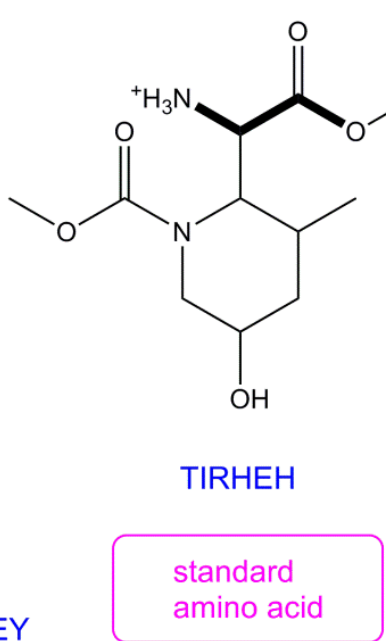




\section{TETRADENTATE}

TRIDENTATE

BIDENTATE ON

BIDENTATE NX'

monodentate

$a(-1)$

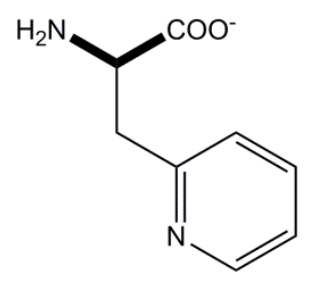

ALTOCO

BAMTUE01

PYALCV
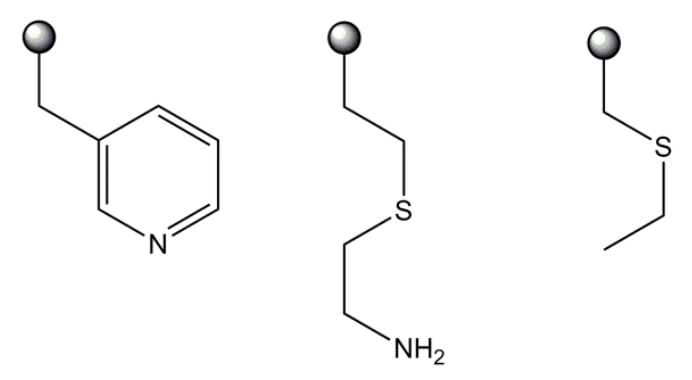

CAPVUK

CEFTAI<smiles>Cc1cn(CO)c(=O)[nH]c1=O</smiles>

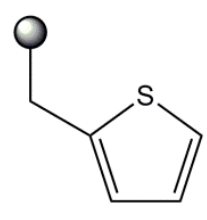

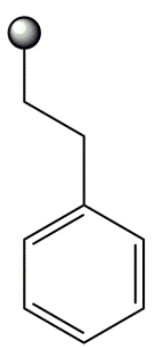

APRCOB

DAPCLC

GAWYOS

JAHNEM

PASKOK

SOPJEN

XAXNOZ

APBMGH

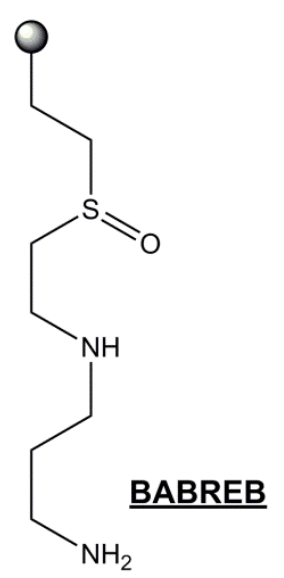

$\underbrace{}_{1}$

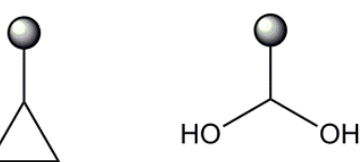

$\overbrace{\mathrm{NH}_{2}}^{\mathrm{N}}$

$\begin{array}{ll} & \text { BAKROV } \\ \text { BAKXER } & \text { BAKRUB }\end{array}$

BUWJIM
CIWLID10 MCYSCD CYSHGA MCYSCO DAPKEM MCYSZN GEVLID WEXYII MCYECO WEXYOO

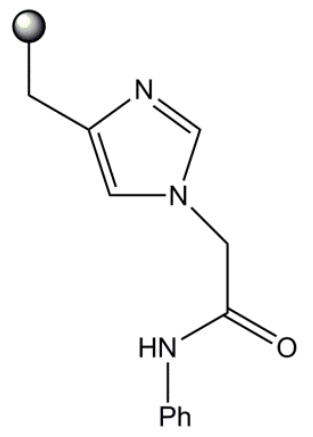

EHUGOD

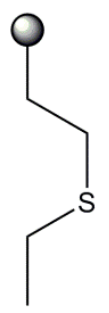

cyshgb

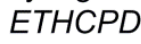

SIXGIP
$\overbrace{\mathrm{NH}_{2}}^{\mathrm{S}}$

FEZFEW

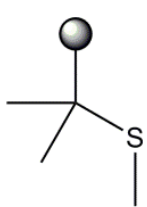

HIGYOL-(2)

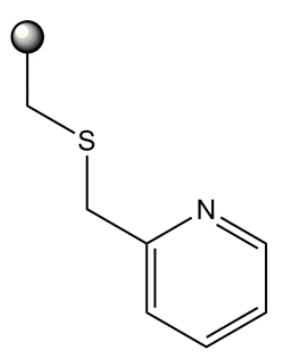

HUQGOQ<smiles>OCc1ccncc1</smiles>

HAWBOY

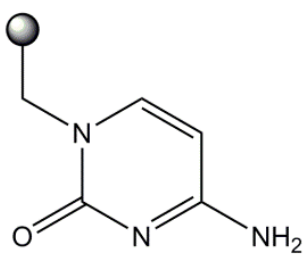

FIZLUW<smiles>OCc1cccc(-c2nnn[nH]2)c1</smiles>

INOXUE INOYAL 
